\title{
Molecular basis for high affinity agonist binding in GPCRs
}

\author{
Tony Warne ${ }^{1}$, Patricia C. Edwards ${ }^{1}$, Andrew S. Doré ${ }^{2}$, Andrew G. W. Leslie ${ }^{1}$, and \\ Christopher G. Tate ${ }^{1,{ }^{*}}$ \\ ${ }^{1} \mathrm{MRC}$ Laboratory of Molecular Biology, Francis Crick Avenue, Cambridge CB2 0QH, UK \\ ${ }^{2}$ Sosei Heptares, Steinmetz Building, Granta Park, Great Abington, Cambridge CB21 6GT, UK
}

\begin{abstract}
G protein-coupled receptors (GPCRs) in the G protein-coupled active state have higher affinity for agonists compared to when they are in the inactive state, but the molecular basis for this is unclear. We have determined four active-state structures of the $\beta_{1}$-adrenoceptor $\left(\beta_{1} \mathrm{AR}\right)$ bound to conformation-specific nanobodies in the presence of agonists of varying efficacy. Comparison with inactive-state structures of $\beta_{1}$ AR bound to the identical ligands showed a $24-42 \%$ reduction in the volume of the orthosteric binding site. Potential hydrogen bonds were also shorter, and there was up to a $30 \%$ increase in the number of atomic contacts between the receptor and ligand. This explains the increase in agonist affinity of GPCRs in the active state for a wide range of structurally distinct agonists.
\end{abstract}

GPCRs exist in an ensemble of conformations that can be selectively stabilized by the binding of a ligand and through interactions with signaling molecules such as $\mathrm{G}$ proteins (1, 2). Pharmacology has characterized at least two distinct states of GPCRs, an active state with high affinity for agonists when coupled to $G$ proteins and an inactive state with low affinity for agonists in the absence of $\mathrm{G}$ proteins (1), although a plethora of sub-states can also exist between these two extremes (3-7). The reason why the active state has a high affinity for agonists is unclear, because receptor structures in the inactive and active states have been determined bound to different ligands, such as for the $\beta_{2}$-adrenoceptor $\left(\beta_{2} \mathrm{AR}\right)$ (8-11). Here we present structures of $\beta_{1} A R$ in the active state and compare them to inactive state structures (12) bound to the identical ligand to define the structural differences in the orthosteric binding site.

"Correspondence to: C.G. Tate, MRC Laboratory of Molecular Biology, Francis Crick Avenue, Cambridge CB2 0QH, UK. cgt@ mrclmb.cam.ac.uk.

Author contributions: T.W. performed receptor and nanobody expression, purification, crystallization, cryo-cooling of the crystals, data collection, data processing and structure refinement. T.W. also performed the pharmacological analyses. P.C.E. purified mini- $\mathrm{G}_{\mathrm{S}}$ and A.D.S assisted with structure solution. A.G.W.L. was involved in data processing and structure solution, refinement and analysis. Manuscript preparation was performed by T.W., A.G.W.L. and C.G.T. The overall project management was by C.G.T.

Competing interests: C.G.T. is a shareholder, consultant and member of the Scientific Advisory Board of Heptares Therapeutics, who also partly funded this work

Data and materials availability: The co-ordinates and structure factors for all the structures determined have been deposited at the PDB with the following accession codes (ligand co-crystallised in parentheses): 6H7J (isoprenaline), 6H7L (dobutamine), 6H7M (salbutamol), 6H7O, cyanopindolol. 
Four crystal structures with overall resolutions between $3.0 \AA$ - $3.2 \AA$ (Table S1) were determined of $\beta_{1} \mathrm{AR}$ bound to either $\mathrm{Nb} 80$ or $\mathrm{Nb6B} 9$, and the overall structures were virtually identical (Fig. 1; 0.2-0.3 $\mathrm{A}$ RMSD for Ca atoms). Nb80 and Nb6B9 are nanobodies originally developed to stabilize the active state of $\beta_{2} \mathrm{AR}(8,10)$ and bind to $\beta_{1} \mathrm{AR}$ due to the high sequence conservation of the receptors. Structures were determined bound to a full agonist (isoprenaline), partial agonists (salbutamol, dobutamine) and a weak partial agonist (cyanopindolol). Isoprenaline, salbutamol and dobutamine showed an increase in affinity when $\beta_{1} A R$ was coupled to the engineered $G$ protein mini- $G_{s}$, whereas cyanopindolol bound with similar high affinity in both the presence and absence of mini- $\mathrm{G}_{\mathrm{s}}$ (Fig. 1). The overall structure of the $\beta_{1}$ AR-nanobody complexes bound to either agonist or partial agonists is virtually identical to that of the agonist-bound Nb6B9- $\beta_{2}$ AR complex $(0.5$ $\AA$ RMSD of 1601 atoms) and the overall conformational changes are virtually identical. These changes result in the partial occlusion of the orthosteric binding pocket (Fig. 2), which is consistent with observations on nanobody-bound $\beta_{2} \mathrm{AR}(8,10)$.

Detailed comparisons were made between the inactive state structures of $\beta_{1} \mathrm{AR}$ with the respective active state structures bound to same ligand (Figs. 2 and 3). In all cases, there was a decrease in the volume (13) of the orthosteric binding site that varied depending on the ligand (Fig 2, Fig. S1). The largest decrease was observed for the full agonist isoprenaline (42\%) and the smallest decrease was observed for the weak partial agonist cyanopindolol $(24 \%)$. The decrease in the volume of the orthosteric binding site when isoprenaline was bound was due primarily to the inward movement of the extracellular ends of $\mathrm{H6}$ and $\mathrm{H} 7$, an inward movement and an increase in the $\mathrm{H} 5$ bulge at Ser $215^{5.46}$ and the reorientation of residues Phe201 ${ }^{\mathrm{ECL} 2}$ and Phe $325^{7.35}$. The magnitude of these changes was greatest for the full agonist isoprenaline and smallest for the weak partial agonist cyanopindolol. The pincerlike movement of Phe201 ${ }^{\mathrm{ECL} 2}$ and Phe $325^{7.35}$ towards the ligand has the largest effect on reducing the volume of the orthosteric binding pocket, with the maximal shift observed in the isoprenaline structure of $3.1 \AA$ for Phe201 ${ }^{\text {ECL2 }}$ and $2.5 \AA$ for Phe $325^{7.35}$ (measured at the $\mathrm{CZ}$ atom of the side chain). The movement of Phe201 ${ }^{\mathrm{ECL} 2}$ appeared to correlate with the structure of the ligand bound, because in all cases it formed van der Waals contacts with the ligand. In contrast, Phe $325^{7.35}$ was not within van der Waals contact with any of the four ligands and moved as a consequence of the inward tilt of $\mathrm{H} 7$.

The reduction in the volume of the orthosteric binding pocket correlated with an overall reduction in the average distance between atoms in the ligand and receptor by $0.1-0.3 \AA$. Amino acid residues in H3, H5, H6, H7 and ECL2 (and H2 for dobutamine) were all involved in contributing to ligand-receptor contacts, but there was no clear pattern to which regions of the receptor changed most significantly (Fig. 3, Fig. S2). Side chains were up to $1.2 \AA$ closer to the ligand in the active state compared to the inactive state. In a number of instances, changes resulted in the strengthening of hydrogen bonds. For example, Asn $310^{6.55}$ was predicted to make a weak hydrogen bond to the para-hydroxyl group of isoprenaline ( $3.5 \AA$ between donor and acceptor) in the inactive state, which changed to 2.8 $\AA$ in the active state. In the active-state structures containing dobutamine and salbutamol, the distance to Ser $215^{5.46}$ was $0.8 \AA$ shorter for both ligands allowing hydrogen bond formation; the hydrogen bond to Ser $211^{5.42}$ also shortened by $0.7 \AA$ to the para hydroxyl in salbutamol, but remained unchanged to the meta hydroxyl in dobutamine. Most of the observed 
differences are due to the contraction of the binding pocket, whereas the significant shortening of the hydrogen bond between Ser2 $11^{5.42}$ and salbutamol is due to a rotamer change. Although all the ligand binding pockets contracted upon receptor activation, the changes in ligand-receptor contacts were not conserved, despite the similarity in chemotypes amongst the four ligands studied. There was a weak correlation between the decrease in volume of the orthosteric binding site and ligand efficacy (Fig. S3), particularly if the most similar chemtoypic ligands were compared (cyanopindolol, salbutamol, isoprenaline). However, there was no correlation between efficacy and the magnitude of ligand affinity increase on receptor activation or the increase in the number of ligand-receptor atomic contacts (Fig. S3). It was particularly notable that cyanopindolol bound to $\beta_{1} \mathrm{AR}$ with similar affinity in both the presence and absence of a coupled G protein (Fig. 1) despite the contraction of the binding pocket and increase in receptor ligand contacts upon activation. This may be a consequence of constraints on the possible conformation change imposed by the rigidity of cyanopindol that prevents the full contraction of the ligand binding pocket by preventing the movement of $\mathrm{H} 7$ and the bulge in $\mathrm{H} 5$ observed in the other structures (Fig. S1).

The role of the partial occlusion of the orthosteric binding site upon activation of $\beta_{1} \mathrm{AR}$ was tested by mutagenesis inspired from the active state structure of $\beta_{2} \mathrm{AR}(8,10)$. In $\beta_{2} \mathrm{AR}$, it was proposed that the occlusion of the binding site was a significant factor in increasing agonist affinity upon $\mathrm{G}$ protein coupling (14). In particular, Tyr308 7.35 was within van der Waals distance of Phe $193^{\mathrm{ECL} 2}$ on the opposite side of the entrance to the orthosteric binding pocket and had a major effect on decreasing the rates of association and dissociation of ligands in the active state compared to the inactive state (14). The $\beta_{2} \mathrm{AR}$ residues

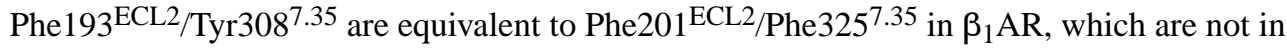
van der Waals contact (Fig. 4). Thus the mutation F325Y 7.35 in $\beta_{1} \mathrm{AR}$ was predicted to occlude the entrance to the orthosteric binding pocket and decrease the rate of ligand association, and conversely, $\mathrm{F} 325 \mathrm{~A}^{7.35}$ was predicted to make the entrance wider and increase the rate of ligand association. When the initial rate of ${ }^{3} \mathrm{H}$-dihydroalprenolol $\left({ }^{3} \mathrm{H}-\right.$ DHA) association was measured (Fig. 4), $\beta_{1} \mathrm{AR}\left(\mathrm{F} 325 \mathrm{~A}\right.$ ) had the same rate as $\beta_{1} \mathrm{AR}$, but $\beta_{1} \mathrm{AR}(\mathrm{F} 325 \mathrm{Y}$ ) had a considerably slower rate of association. However, the affinities (Fig. 4) of epinephrine and isoprenaline for the high affinity state of $\beta_{1} A R$ and $\beta_{1} A R(F 325 Y)$ were identical and there was only a minor difference with norepinephrine. Comparisons of affinities (Fig. 4) for the inactive states of $\beta_{1} A R$ and $\beta_{1} \mathrm{AR}(\mathrm{F} 325 \mathrm{Y})$ showed a large decrease in affinities for norepinephrine (14.8-fold), epinephrine (11.2-fold) and isoprenaline (7.6fold), which implied that the greater agonist affinity shift observed in $\beta_{1} \mathrm{AR}(\mathrm{F} 325 \mathrm{Y})$ compared to $\beta_{1} \mathrm{AR}$ was due to destabilisation of the inactive state and not stabilisation of the active state. This suggested that partial occlusion of the ligand binding pocket in $\beta_{1} \mathrm{AR}(\mathrm{F} 325 \mathrm{Y})$ during formation of the active state played little role in the increase of agonist affinity on $\mathrm{G}$ protein coupling.

The destabilising effect of the F325Y mutation in $\beta_{1} \mathrm{AR}$ on the agonist-bound inactive state suggested that converting the extracellular surface of $\beta_{2} \mathrm{AR}$ to make it similar to $\beta_{1} \mathrm{AR}$ would increase the affinity of the inactive state and leave the affinity of the $G$ proteincoupled activated state approximately unchanged. The $\beta_{2} \mathrm{AR}$ mutant constructed, $\beta_{2} \mathrm{AR}\left(\beta_{1} \mathrm{LBP}\right)$, did indeed show these characteristics (Fig S4; see Methods for the rationale 


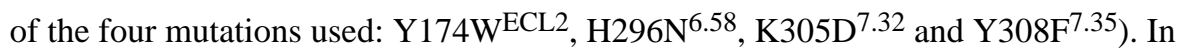
addition, the accessibility of the $\beta_{1}$ AR orthosteric binding pocket in the $G$ protein-coupled state to ${ }^{125} \mathrm{I}$-cyanopindolol was greater than that observed for $\beta_{2} \mathrm{AR}$ (Fig. S5). The four mutations in $\beta_{2} \mathrm{AR}\left(\beta_{1} \mathrm{LBP}\right)$ converted the behaviour of $\beta_{2} \mathrm{AR}$ to that of $\beta_{1} \mathrm{AR}$. Adding the converse residues from $\beta_{2} \mathrm{AR}$ into $\beta_{1} \mathrm{AR}$, to make the mutant $\beta_{1} \mathrm{AR}\left(\beta_{2} \mathrm{LBP}\right)$, converted the accessibility of the orthosteric binding site in $\beta_{1}$ AR to that of $\beta_{2}$ AR (Fig. S5).

The increase in affinity of agonist binding to the active state of $\beta_{1} \mathrm{AR}$ arises from the increase in the number and/or strength of ligand-receptor contacts (a thermodynamic effect). This is consequently associated with a decrease in the rate of ligand dissociation. Where a native ligand is a large peptide that interacts with extracellular domains, this thermodynamic effect may be the major contributor to the decrease in the rate of ligand dissociation. However, where the ligand is small, such as adrenaline or acetylcholine, the rate of ligand dissociation may also be decreased due to a purely steric blockage of the entrance to the ligand binding pocket (a kinetic effect) (14). Given the high conservation of both the structure and function of GPCRs $(15,16)$, these considerations are likely to apply to other GPCRs that bind diffusible ligands.

\section{Supplementary Material}

Refer to Web version on PubMed Central for supplementary material.

\section{Acknowledgments}

We thank the beamline staff at the European Synchrotron Radiation Facility (beamlines ID23-2, ID30-A3, ID29, ID30B and MASSIF-1) and at Diamond Light Source (beamline I24).

Funding: This work was supported by core funding from the Medical Research Council [MRC U105197215 and U105184325] and a grant from the ERC (EMPSI 339995)

\section{References and Notes}

1. Gether U. Uncovering molecular mechanisms involved in activation of G protein-coupled receptors. Endocr Rev. 2000; 21:90-113. [PubMed: 10696571]

2. Rosenbaum DM, Rasmussen SG, Kobilka BK. The structure and function of G-protein-coupled receptors. Nature. 2009; 459:356-363. [PubMed: 19458711]

3. Vauquelin G, Van Liefde I. G protein-coupled receptors: a count of 1001 conformations. Fundam Clin Pharmacol. 2005; 19:45-56. [PubMed: 15660959]

4. Gregorio GG, et al. Single-molecule analysis of ligand efficacy in beta2AR-G-protein activation. Nature. 2017; 547:68-73. [PubMed: 28607487]

5. Yao XJ, et al. The effect of ligand efficacy on the formation and stability of a GPCR-G protein complex. Proc Natl Acad Sci U S A. 2009; 106:9501-9506. [PubMed: 19470481]

6. Manglik A, et al. Structural Insights into the Dynamic Process of beta2-Adrenergic Receptor Signaling. Cell. 2015; 161:1101-1111. [PubMed: 25981665]

7. Ye L, Van Eps N, Zimmer M, Ernst OP, Prosser RS. Activation of the A2A adenosine G-proteincoupled receptor by conformational selection. Nature. 2016; 533:265-268. [PubMed: 27144352]

8. Rasmussen SG, et al. Structure of a nanobody-stabilized active state of the beta(2) adrenoceptor. Nature. 2011; 469:175-180. [PubMed: 21228869]

9. Rasmussen SG, et al. Crystal structure of the beta2 adrenergic receptor-Gs protein complex. Nature. 2011; 477:549-555. [PubMed: 21772288] 
10. Ring AM, et al. Adrenaline-activated structure of beta2-adrenoceptor stabilized by an engineered nanobody. Nature. 2013; 502:575-579. [PubMed: 24056936]

11. Rosenbaum DM, et al. Structure and function of an irreversible agonist-beta(2) adrenoceptor complex. Nature. 2011; 469:236-240. [PubMed: 21228876]

12. Warne $\mathrm{T}$, et al. The structural basis for agonist and partial agonist action on a beta(1)-adrenergic receptor. Nature. 2011; 469:241-244. [PubMed: 21228877]

13. Durrant JD, Votapka L, Sorensen J, Amaro RE. POVME 2.0: An Enhanced Tool for Determining Pocket Shape and Volume Characteristics. J Chem Theory Comput. 2014; 10:5047-5056. [PubMed: 25400521]

14. DeVree BT, et al. Allosteric coupling from $\mathrm{G}$ protein to the agonist-binding pocket in GPCRs. Nature. 2016; 535:182-186. [PubMed: 27362234]

15. Venkatakrishnan AJ, et al. Diverse activation pathways in class A GPCRs converge near the Gprotein-coupling region. Nature. 2016; 536:484-487. [PubMed: 27525504]

16. Venkatakrishnan AJ, et al. Molecular signatures of G-protein-coupled receptors. Nature. 2013; 494:185-194. [PubMed: 23407534]

17. Carpenter B, Tate CG. Engineering a minimal G Protein to facilitate crystallisation of G protein coupled receptors in their active conformation. Protein Eng Design Sel. 2016; 29:583-593.

18. Warne T, Chirnside J, Schertler GF. Expression and purification of truncated, non-glycosylated turkey beta-adrenergic receptors for crystallization. Biochim Biophys Acta. 2003; 1610:140.

19. Warne T, Serrano-Vega MJ, Tate CG, Schertler GF. Development and crystallization of a minimal thermostabilised G protein-coupled receptor. Protein Expr Purif. 2009; 65:204-213. [PubMed: 19297694]

20. Urbani A, Warne T. A colorimetric determination for glycosidic and bile salt-based detergents: applications in membrane protein research. Anal Biochem. 2005; 336:117-124. [PubMed: 15582566]

21. Battye TG, Kontogiannis L, Johnson O, Powell HR, Leslie AG. iMOSFLM: a new graphical interface for diffraction-image processing with MOSFLM. Acta Crystallogr D Biol Crystallogr. 2011; 67:271-281. [PubMed: 21460445]

22. Evans PR, Murshudov GN. How good are my data and what is the resolution? Acta Crystallogr D Biol Crystallogr. 2013; 69:1204-1214. [PubMed: 23793146]

23. McCoy AJ, et al. Phaser crystallographic software. Journal of applied crystallography. 2007; 40:658-674. [PubMed: 19461840]

24. Murshudov GN, et al. REFMAC5 for the refinement of macromolecular crystal structures. Acta Crystallogr D Biol Crystallogr. 2011; 67:355-367. [PubMed: 21460454]

25. Emsley P, Cowtan K. Coot: model-building tools for molecular graphics. Acta Crystallogr D Biol Crystallogr. 2004; 60:2126-2132. [PubMed: 15572765]

26. Carpenter B, Tate CG. Expression and Purification of Mini G Proteins from Escherichia coli. Bio Protoc. 2017; 7

27. Baker JG, Proudman RG, Hill SJ. Identification of key residues in transmembrane 4 responsible for the secondary, low-affinity conformation of the human beta1-adrenoceptor. Mol Pharmacol. 2014; 85:811-829. [PubMed: 24608857]

28. Baker JG, Proudman RG, Tate CG. The pharmacological effects of the thermostabilising (m23) mutations and intra and extracellular (beta36) deletions essential for crystallisation of the turkey beta-adrenoceptor. Naunyn Schmiedebergs Arch Pharmacol. 2011; 384:71-91. [PubMed: 21547538] 


\section{One Sentence Summary}

High affinity agonist binding to G protein-coupled GPCRs results from an increase in the number and strength of protein-ligand interactions. 


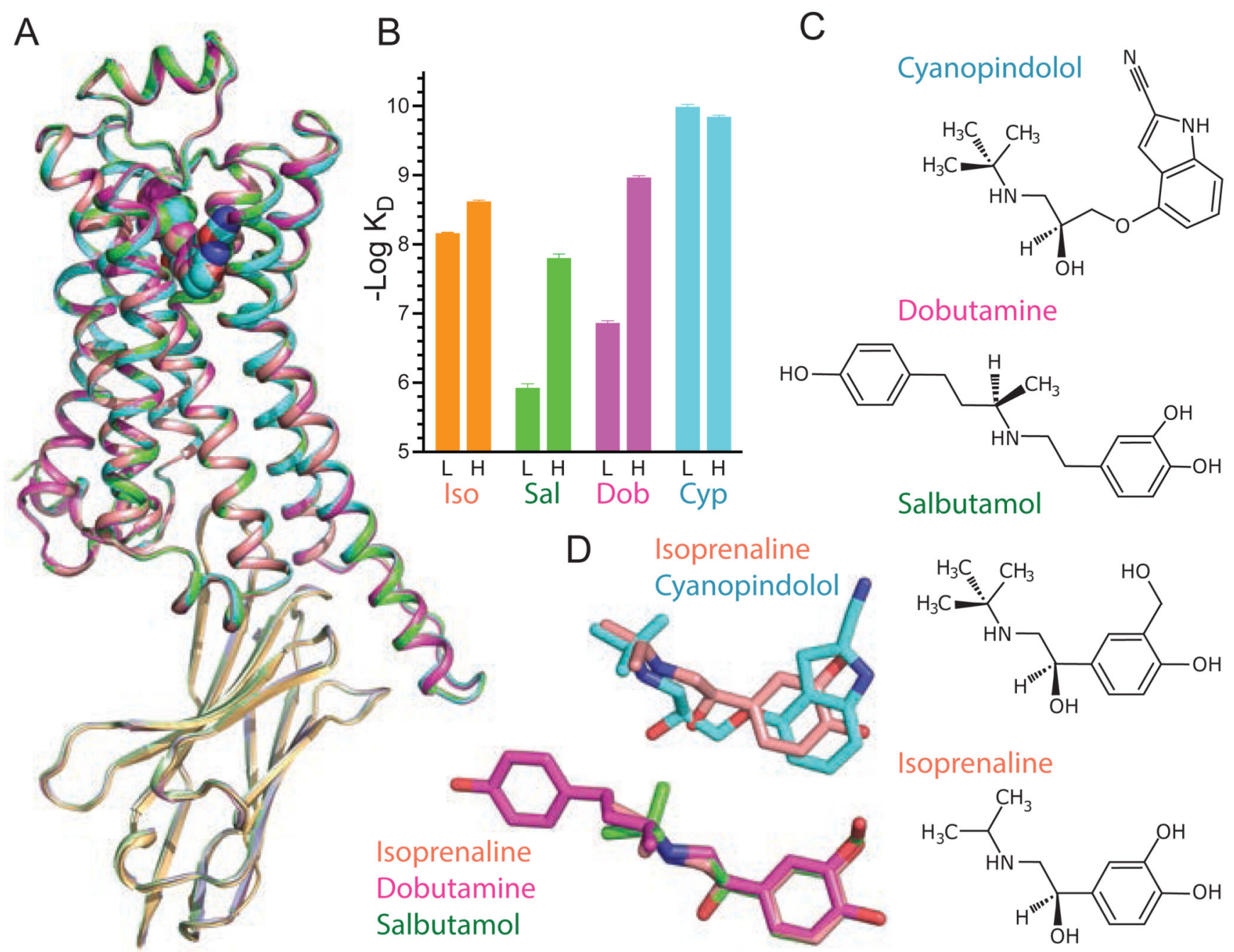

Fig. 1. Structure of the active state of agonist-bound $\beta_{1} A R$-nanobody complex.

(A) Superposition of four structures of $\beta_{1}$ AR-nanobody complexes bound to ligands shown in (C). (B) Affinities of $\beta_{1} A R$ in the low affinity state, $L$, and high affinity state coupled to mini- $\mathrm{G}_{\mathrm{S}}, \mathrm{H}$, for the ligands co-crystallised with the receptor. Data are in Tables S2 and S3, and Fig. S6. Results are the mean of 2-4 experiments performed in duplicate with error bars representing the SEM. (C) Structures of the ligands co-crystallised in the $\beta_{1}$ AR complexes. (D) Disposition of the ligands after superposition of the receptors, using the same colour coding as in (B). See Fig. S9 for ligand densities. 

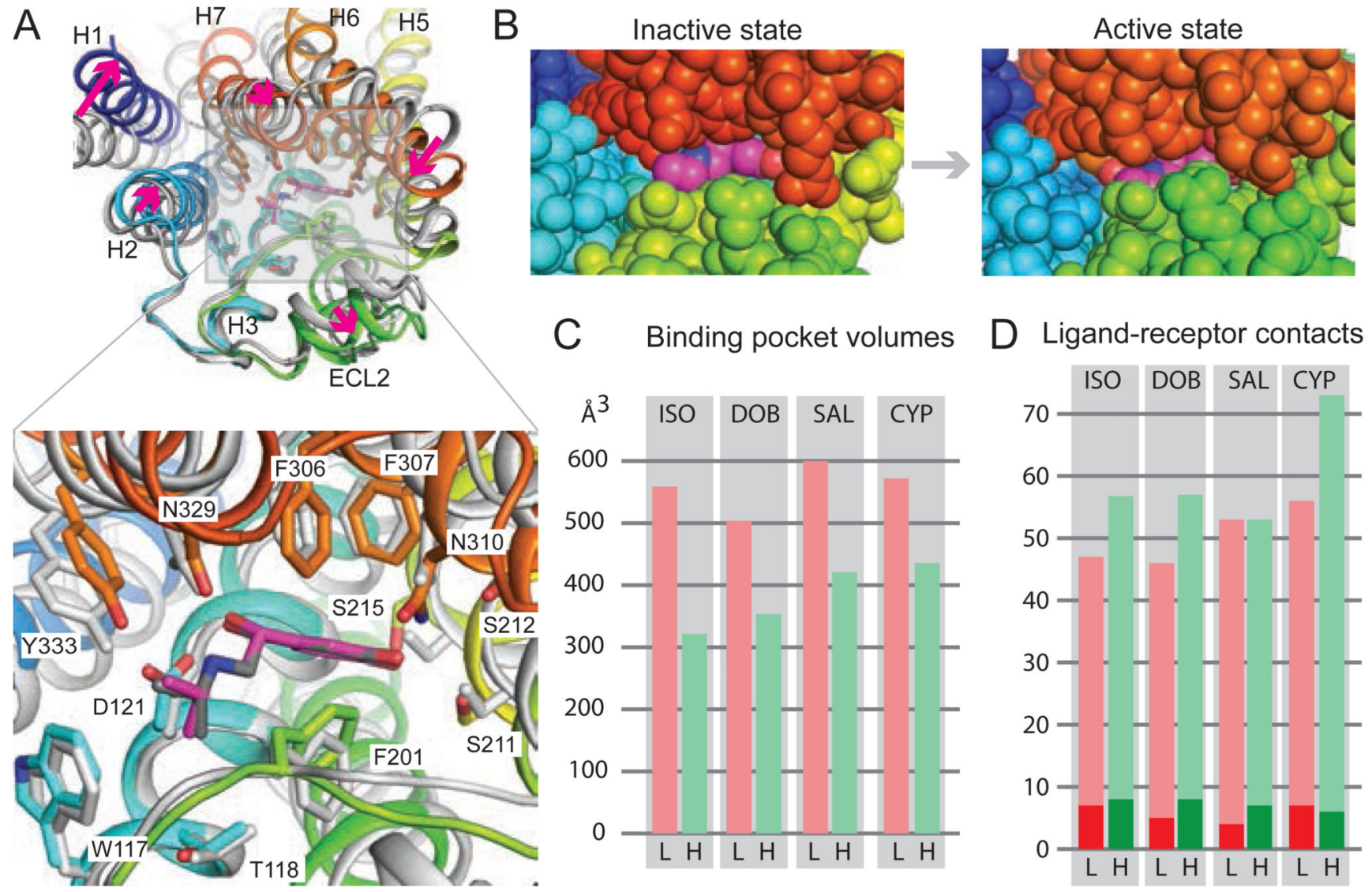

Fig. 2. Conformational changes in isoprenaline-bound $\beta_{1} A R$.

(A), Superposition of isoprenaline-bound $\beta_{1} A R$ in the inactive state (grey, PDB ID 2Y03) with isoprenaline-bound $\beta_{1} \mathrm{AR}$ in the active state (rainbow colouration). Arrows (magenta) indicate the transitions from the inactive to active state. Alignment was performed based on the isoprenaline molecules using PyMol (magenta, isoprenaline bound to active state $\beta_{1} \mathrm{AR}$ ). (B) View of the orthosteric binding site from the extracellular surface with atoms shown as space filling models: isoprenaline (magenta, carbon atoms); $\beta_{1} \mathrm{AR}: \mathrm{H} 1$, dark blue; $\mathrm{H} 2$, light blue; H5, yellow; ECL2, green; ECL3 and parts of H6 and H7, red. (C) Volumes of the orthosteric binding site in the low-affinity inactive state (L, pink bars) compared to the highaffinity active state (H, green bars). (D) Number of atomic contacts (Database S1) between the respective ligands and $\beta_{1} \mathrm{AR}$ in the low-affinity inactive state (L, pink bars) compared to the high-affinity active state $(\mathrm{H}$, green bars). The dark shades represent the number of polar interactions. Ligand abbreviations are shown in Fig. 1. 
Isoprenaline
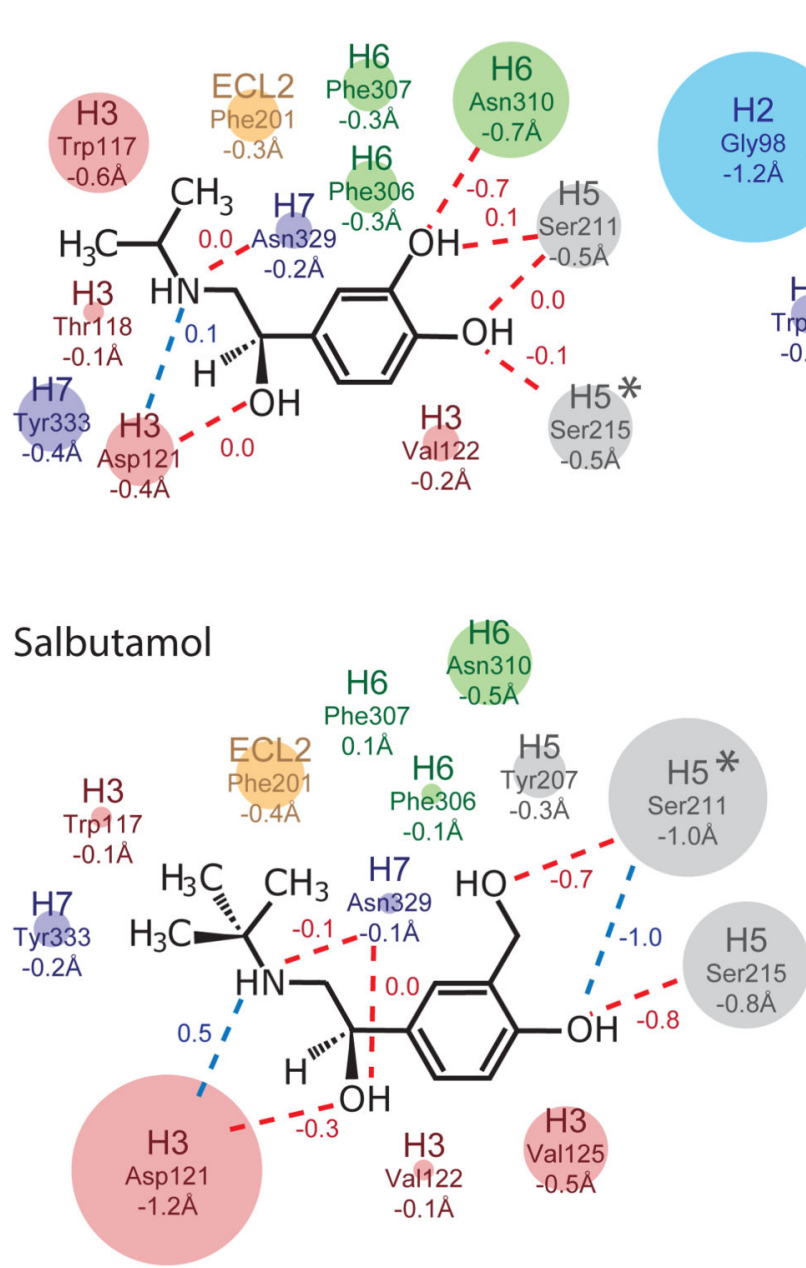

Dobutamine

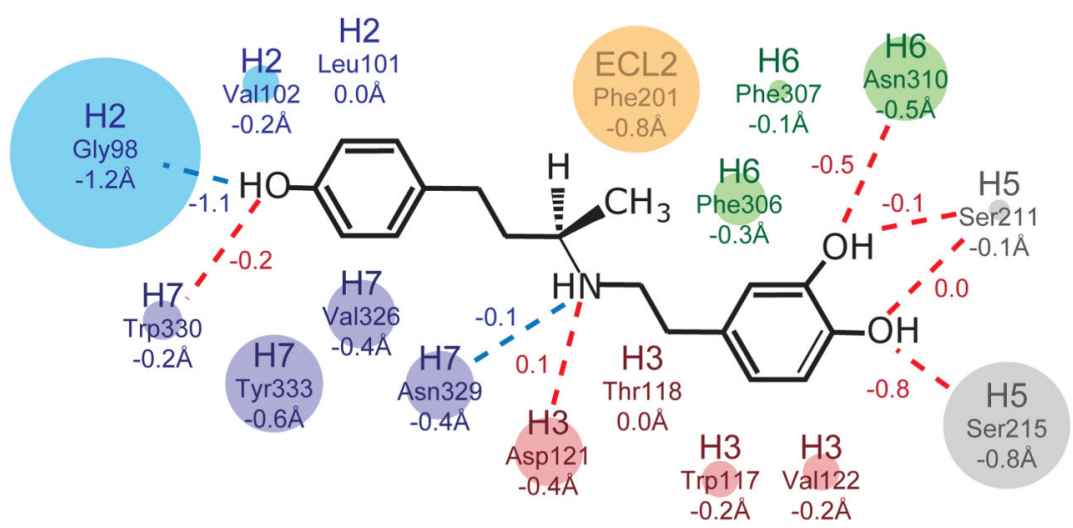

Cyanopindolol
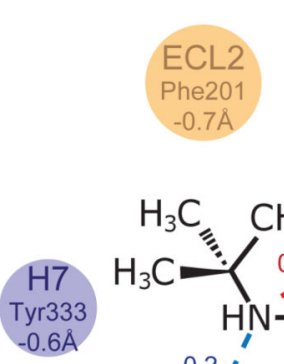

Phe201

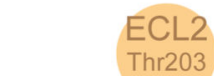

ECL2
Thr203

$\begin{array}{cr}\mathrm{H} 6 & -0.2 \text { I } \\ \text { Phe307 } & -0.1 \AA \text { H6 } \\ & \text { N }\end{array}$

$2^{1}$

$\mathrm{H} 5$
$\mathrm{H}$ Ala208

Phe 306
$-0.4 \AA$

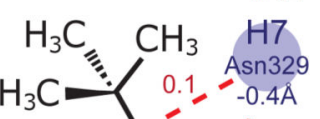

$\mathrm{H}_{3} \mathrm{C}=\begin{array}{cc}\mathrm{CH}_{3} & \mathrm{H} 7 \\ 0.1 & -{ }_{-0.4 \AA}\end{array}$

$\mathrm{NHH}_{-0.2}^{--}$

$0.0 \AA$ Tyr207

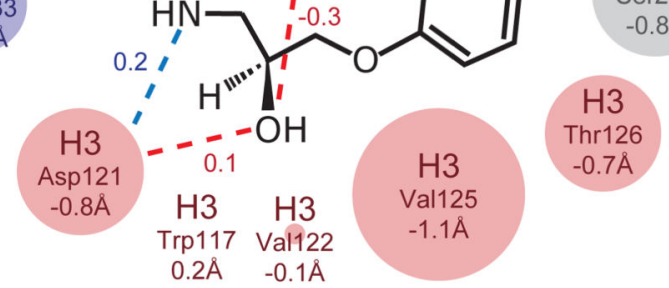

Fig. 3. Changes in $\boldsymbol{\beta}_{\mathbf{1}} \mathrm{AR}$-ligand contact distances.

The maximal changes in contact distances between ligands and atoms in $\beta_{1} \mathrm{AR}$ from the inactive to active states are depicted. Amino acid side chains making contact to the ligands are indicated and coloured according to where they are in $\beta_{1} \mathrm{AR}$ (blue, H2; red, H3; orange, ECL2; grey, H5; green, H6; purple, H7) with the diameter of the circle representing the magnitude of the distance change (shown as numbers below the amino acid residue).

Numbers next to the lines indicate the change in length of polar contacts (blue dashed lines) and hydrogen bonds (red dashed lines; determined using HBPLUS). Negative numbers imply a decrease in distance between the ligand and receptor in the transition from the inactive state to the active state. An asterisk indicates a significant rotamer change between the inactive and active states. For the details of additional contacts made by each side chain, see Fig. S2. 


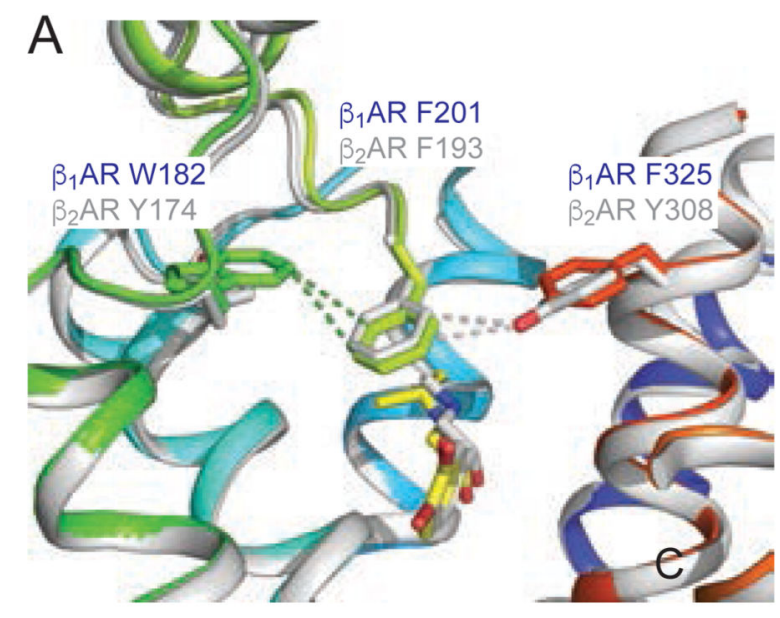

C

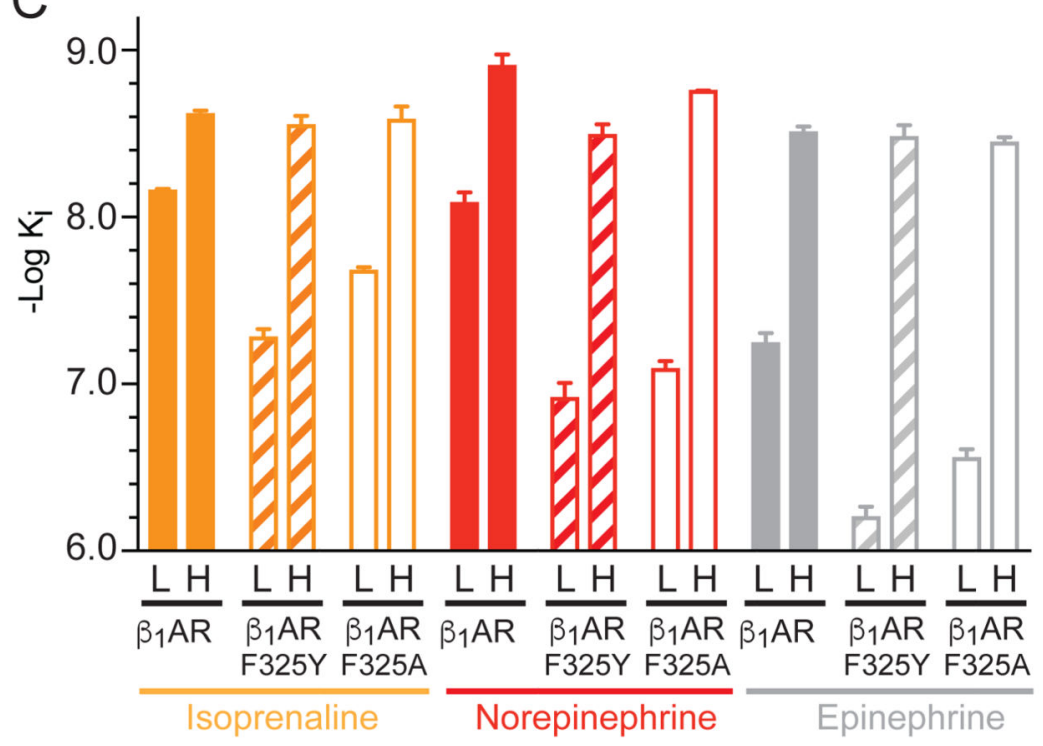

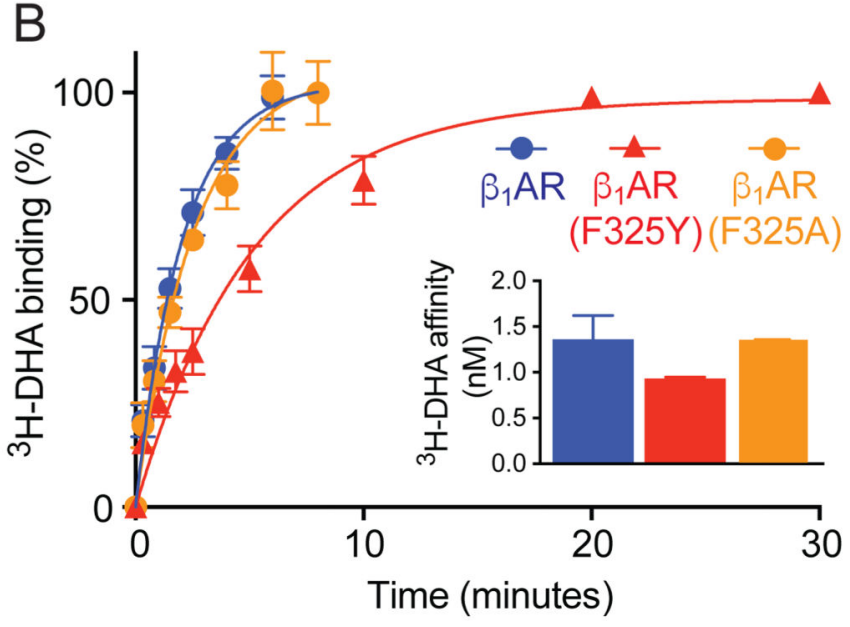

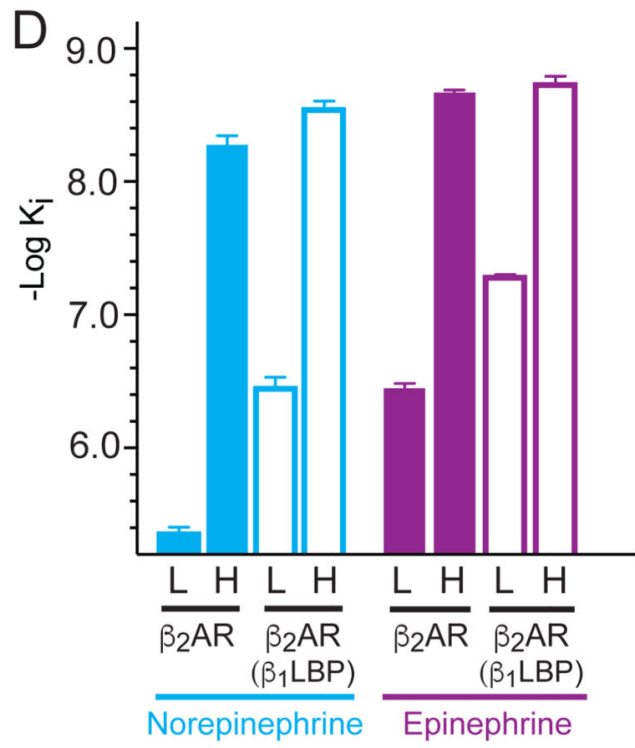

Fig. 4. Comparisons between $\beta_{1} A R$ and $\beta_{2} A R$.

(A) Alignment of the active state structures of $\beta_{1} \mathrm{AR}$ (rainbow colouration) and $\beta_{2} \mathrm{AR}$ (grey, PDB ID 4LDO). Ligands are shown as sticks; isoprenaline, yellow; adrenaline, grey. (B) Rate of association of the radioligand ${ }^{3} \mathrm{H}-\mathrm{DHA}$ on to $\beta_{1} \mathrm{AR}$ (blue circles), $\beta_{1} \mathrm{AR}(\mathrm{F} 325 \mathrm{Y})$ (red triangles) and $\beta_{1} \mathrm{AR}(\mathrm{F} 325 \mathrm{~A})$ (orange circles). The inset shows the affinities of ${ }^{3} \mathrm{H}-\mathrm{DHA}$ (same colour code). (C, D) Affinities of $\beta_{1} A R, \beta_{2} A R$ and their respective mutants in the low affinity state, $\mathrm{L}$, and high affinity state coupled to mini- $\mathrm{G}_{\mathrm{s}}, \mathrm{H}$. All data are in Tables S2 and S3 and representative graphs of affinity shifts are in Figure S6. Results are the mean of 2-6 experiments performed in duplicate with error bars representing the SEM. 